\title{
LncRNA SNHG16 promotes cell proliferation through miR-302a-3p/FGF19 axis in hepatocellular carcinoma
}

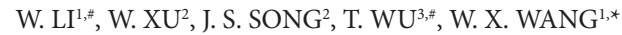 \\ ${ }^{1}$ Department of General Surgery, Renmin Hospital of Wuhan University, Wuhan, 430060, Hubei, China; ${ }^{2}$ Department of General Surgery, First \\ People's Hospital of Tianmen, Tianmen, 431700, Hubei, China; ${ }^{3}$ Department of Ultrasound Imaging, Renmin Hospital of Wuhan University, \\ Wuhan, 430060, Hubei, China
}

${ }^{*}$ Correspondence: sate.llite@163.com

${ }^{*}$ Contributed equally to this work.

Received July 20, 2018 / Accepted November 21, 2018

\begin{abstract}
Hepatocellular carcinoma (HCC) is the sixth most common malignancy worldwide and the leading cause of death in Asian and African countries. Aberrant accumulation of lncRNAs is one of the major causes of tumorigenesis in HCC. Small nucleolar RNA host gene 16 (SNHG16) is identified as an oncogene in multiple types of tumors. However, the role of SNHG16 in HCC is poorly understood. Herein, we show that SNHG16 is up-regulated and associated with poor prognosis in HCC. We also demonstrate that SNHG16 interacts with miR-302a-3p and decreases its expression. Moreover, our results indicate that SNHG16/miR-302a-3p axis regulates expression of the FGF19 in liver cancer cells. Finally, we investigated the biological function of SNHG16 in HCC and showed that SNHG16 promotes liver cancer cell proliferation via the SNHG16/ miR-302a-3p/FGF19 axis. Collectively, these data suggest that SNHG16 might be a predictive biomarker and a potential therapeutic target in liver cancer.
\end{abstract}

Key words: SNHG16, miR-302a-3p, FGF19, hepatocellular carcinoma, cell proliferation

Hepatocellular carcinoma (HCC) is the sixth common malignancies worldwide and the leading cause of death in Asian and African countries with a higher rate of $\mathrm{HBV}$ and HCV infections [1]. Although surgical treatment and chemotherapy are proved to be effective for treatment of HCC patients, there are still a large number of patients who are hard to benefit from the current treatment. Therefore, exploring of the molecular mechanism underlying HCC is critical for the development of effective treatment strategies.

Long non-coding RNAs (lncRNAs) are more than 200 nucleotides in length and belong to the non-coding RNA family [2]. LncRNAs occupy a large proportion of transcriptomes in cells and play important roles in cell growth, activation, apoptosis and differentiation [2]. Aberrant accumulation of lncRNAs is one of major causes of tumorigenesis in multiple types of solid tumor, including HCC $[2,3]$. Small nucleolar RNA host gene 16 (SNHG16) localized at 17q25.1 is originally identified as an oncogene in neuroblastoma [4], bladder cancer [5] and colorectal cancer [6]. The expression of SNHG16 is reported to be linked with poor prognosis in neuroblastoma, bladder cancer and colorectal cancer patients. SNHG16 exhibited oncogenic phenotypes by promoting cell proliferation in colorectal cancer, bladder cancer and neuroblastoma cell lines. Reduced SNHG16 expression increased chemotherapy sensitivity in bladder cancer cells lines [5].

The main objectives of the current study were to investigate the role of SHNG16 in HCC. In our study, we examined the clinical relevance of SNHG16 in HCC and found that overexpressed SNHG16 was associated with poor prognosis in HCC specimens. Then, we showed that SNHG16 interacted with miR-302a-3p and depressed its expression. Moreover, we demonstrated that SNHG16/miR-302a-3p axis regulated the expression of FGF19 in liver cancer cells. Finally, we investigated the biological function of SNHG16 in HCC and showed that SNHG16 promoted liver cancer cells proliferation through the SNHG16/miR-302a-3p/FGF19 axis.

\section{Materials and methods}

Cell culture and tissue samples. All liver cancer cell lines, including Huh7, HepG2, SMMC7721, SK-Hep1 and Hep 3B, and human fetal hepatocyte line LO2 were purchased from the Chinese Academy of Science Cell Bank. All cells were grown in the DMEM medium (Gibco, USA) with 10\% fetal 
bovine serum. The cell lines were maintained in a humidified atmosphere at $37^{\circ} \mathrm{C}, 5 \% \mathrm{CO}_{2}$. All of HCC tissues samples $(\mathrm{n}=34)$ were obtained during operations at our hospital. All of HCC patients $(n=34)$ signed informed consent and this study was approved by the Ethics Committee of First People's hospital of Tianmen.

Cell transfection. The miR mimic and inhibitor used in this study were purchased from Gene Pharma Co., Ltd. (Shanghai, China). Transfections were conducted using lipid-based method (Lipofectamine 2000, Thermo Fisher Scientific) following the manufacturer's instructions.

Real-time RT-PCR. Total RNA was extracted from cells and tissue samples using Trizol reagent (Thermo Fisher Scientific). $1 \mu \mathrm{g}$ RNA was reversely transcribed into first-strand cDNA by using a cDNA Reverse Transcription kit and real-time PCR analysis was carried out with a PCR kit according to the manufacturer's protocols. The two kits were purchased from Takara Bio Inc. (Shigo, Japan). All signals were normalized against GAPDH and the $2^{-\Delta \Delta \mathrm{Ct}}$ method was used to quantify the fold change. Primers used: SNHG16-F: AAGACATGGCCACTCCAGTC; SNHG16-R: TCGTCACCACTTCGTCTCTG; miR-302a-3p-F: ATGAGCTGCGGTCAATACAA; miR-302a-3p-R: GGATTCTAACTTTTTCCAAGACTGG; FGF19-F: GCACAGTTTGCTGGAGATCA; FGF19-R: ATCTCCTCCTCGAAAGCACA; GAPDH-F: GGATTTGGTCGTATTGGG; GAPDH-R: GGAAGATGGTGATGGGATT.

Luciferase assay. SNHG16 and FGF19 3'-untranslated region (UTR) containing the putative miR-302a-3p binding site were cloned into a pMIR-REPORT plasmids (Promega) to form the reporter vector pMIR-SNHG16-WT and FGF19WT. GeneArt ${ }^{\text {tw }}$ Site-Directed Mutagenesis System (Thermo Fisher Scientific) was used to produce mutant-type SNHG16 reporter (SNHG16-Mut) and mutant-type FGF19 reporter (FGF19-Mut). SNHG16-WT and Mut or FGF19-WT and Mut were co-transfected with miR-302a-3p mimics into cells. Luciferase activities were determined using a dualluciferase assay system (Promega) following the protocols of the manufacturer.

Biotin pull-down assay. HepG2 and Huh7 cells were plated in the 6-well plate. Cells were transfected with Bio-SNHG20, or negative control using Lipofectamine 2000. After $48 \mathrm{~h}$, cells were harvested and lysed. The level of miR-302a-3p in the pull-down of Bio-SNHG16 or negative control was quantified by qRT-PCR.

Western blotting. Cells were collected by centrifugation and lysed using buffer for Western blotting (Beyotime, China, P0013). Protein concentrations were determined using the BCA method. Proteins were heating at $100^{\circ} \mathrm{C}$ for 5 minutes in sample buffer. Then protein were separated on SDS-PAGE gels and transferred onto PVDF membranes (Pierce Biotechnology). The membranes were blocked in 5\% not-fat milk for 1 hour at room temperature and incubated with a primary antibody overnight at $4{ }^{\circ} \mathrm{C}$. The membranes were then washed with 1xTBST and incubated with a secondary antibody for 1 hour. Finally, the membranes were visualized by chemiluminescence. The primary antibody used as follows: FGF19 (Abcam, ab85042, dilution 1:1000), Tubulin (Cell signal technology, 5346, dilution 1:1000).

MTS assay. Cell viability was determined using a CellTiter $96^{\circ}$ AQueous One Solution Cell Proliferation Assay kit (Promega) accord-ing to the manufacturer's instructions. Generally, at the indicated time points (1,2,3, and 4 day) after transfection, cells were incubated with Cell-Titer 96 AQueous One Solution reagent for $1 \mathrm{~h}$. Then, cell absorbance was measured at $490 \mathrm{~nm}$.

Statistical analysis. Statistical analyses were performed with one-sided or two-sided paired Student $t$ test for single comparison and One-way ANOVA and a post hoc test for multiple comparisons. A p-value $<0.05$ was considered statistically significant. All values were expressed as means \pm SD.

\section{Results}

LncRNA SNHG16 is upregulated and associated with poor prognosis in Hepatocellular carcinoma. Given that lncRNAs are critical for the tumorigenesis [1], the roles of lncRNAs in hepatocellular carcinoma (HCC) development have risen up. To investigate the specific role of SNHG16 in HCC progression, we firstly examined SNHG16 mRNA level in HCC and normal liver tissues. We found that SNHG16 was overexpressed in HCC compared with normal liver tissues (Figure 1A). Next, we checked the SNHG16 in liver cancer cell lines. In consistent with the finding in HCC patient specimens, the expression of SNHG16 in liver cancer cell lines was higher than normal liver cell lines (Figure 1B). Moreover, we analyzed the disease free survival rate and overall survival rate in HCC patients using GEPIA web tool [7]. Results indicated that SNHG16 in HCC was significantly associated with poor disease free survival and overall survival of patients (Figures 1C, 1D). Together, these data indicate that SNHG16 is up-regulated and associated with poor prognosis in HCC specimens.

SNHG16 interacts with miR-302a-3p and depresses its expression in HCC. As up-regulated SNHG16 linked to poor prognosis in HCC, we want to search the specific molecular mechanism of SNHG16 in HCC. It has been documented that lncRNAs could function as miRNA sponges and inhibit the expression of miRNA via binding with the mRNA of miRNA [8]. To investigate whether SNHG16 interacted with miRNA, we found that SNHG16 possessed a potential binding site of miR-302a-3p using Starbase web tool [9] (Figure 2A). Next, to examine whether SNHG16 repressed the expression of miR-302a-3p in HCC, we knocked down the SNHG16 in both HepG2 and Huh7 liver cancer cell lines (Figure 2B). Our data demonstrated that knockdown of SNHG16 increased miR-302a-3p expression in liver cancer cell lines (Figure 2B). To confirm the interaction between miR-302a-3p and SNHG16, we performed dual-luciferase reporter assay and found that luciferase activity was remarkably decreased in cells co-transfected with SNHG16-Wt and miR-302a-3p 
A

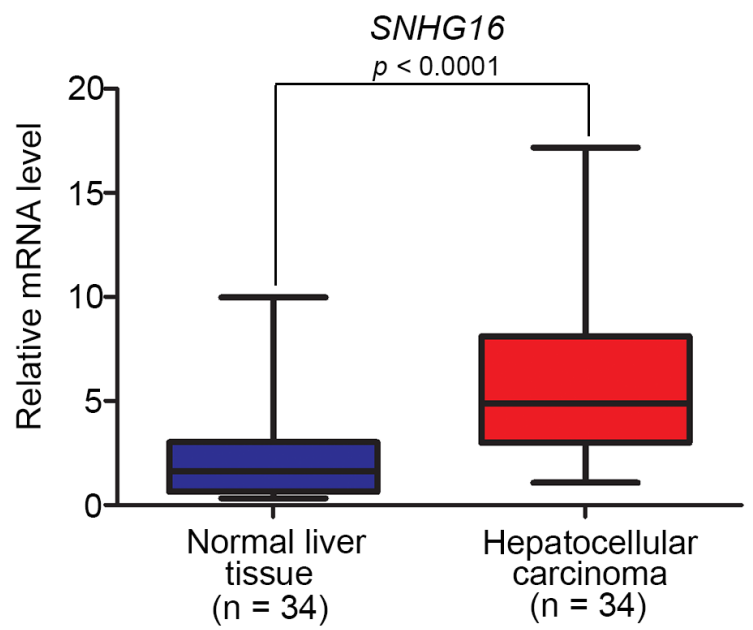

C

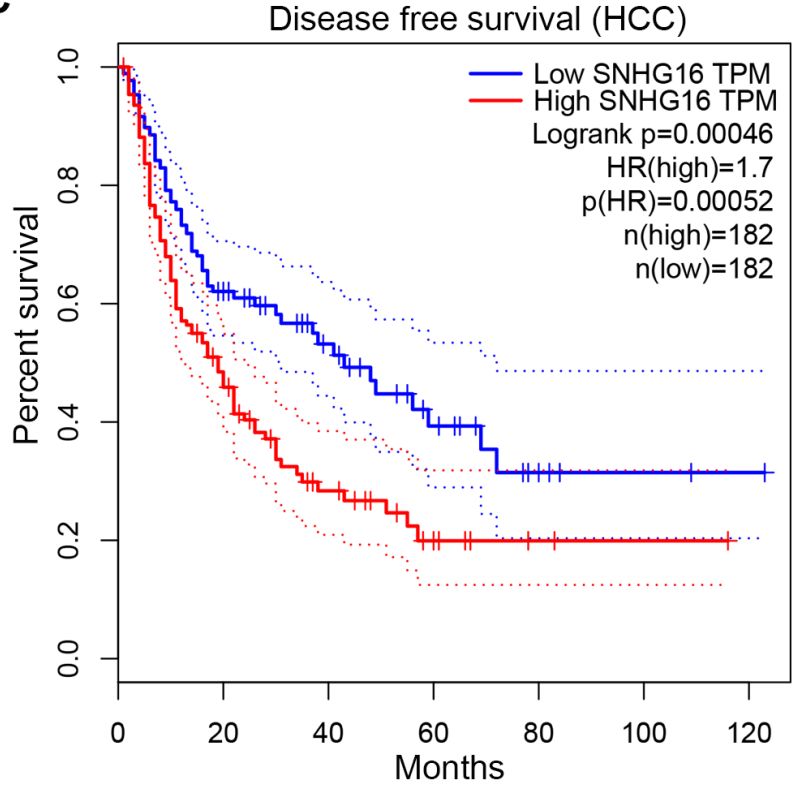

B

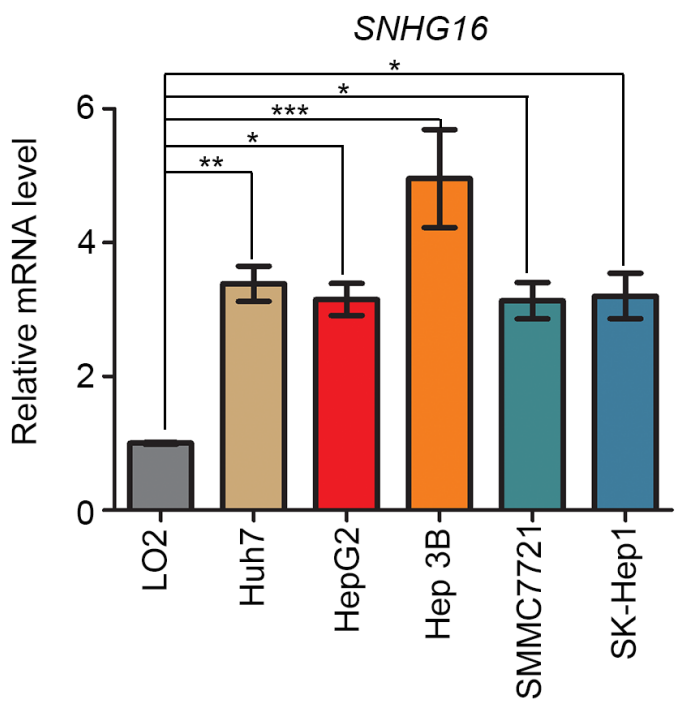

D

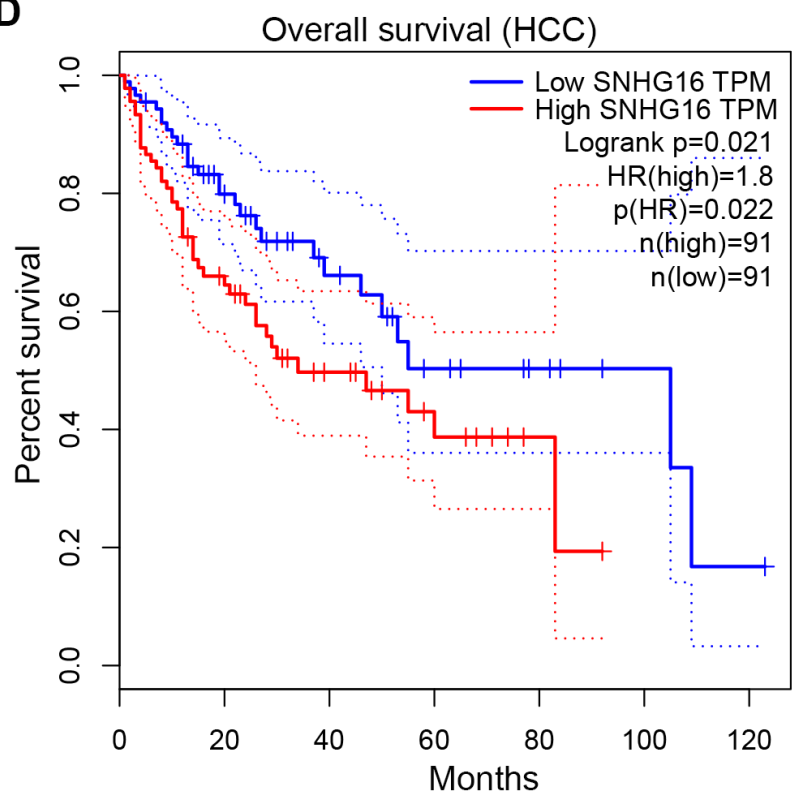

Figure 1. LncRNA SNHG16 is upregulated and associated with poor prognosis in hepatocellular carcinoma. A) SNHG16 expression was upregulated in liver cancer tissues compared with normal liver tissue $(n=34)$. B) RT-qPCR analysis of SNHG16 expression level in indicated cell lines. Data shown are mean values \pm SD from three replicates. ${ }^{\star} \mathrm{p}<0.05 ;{ }^{* *} \mathrm{p}<0.01 ;{ }^{\star * *} \mathrm{p}<0.001$. The disease-free (C) and overall survival (D) rate of liver cancer patients with low or high expression of SNHG16 were computed by GEPIA dataset..

mimics (Figure 2C). Moreover, biotin pull down assay indicated that the miR-302a-3p obtained a great enrichment in the SNHG16 pulled down pellets compared with negative control in both HepG2 and Huh7 cells (Figure 2D). Furthermore, we detected the expression of miR-302a-3p in HCC and normal liver tissues. Results showed that miR-302a-3p was down-regulated in HCC compared to normal liver tissue (Figure 2D). Statistical analysis revealed that the SNHG16 expression was inversely correlated with miR-302a-3p in this cohort (Pearson's product-moment correlation $r^{2}=0.407$, $\mathrm{p}<0.05$, Figure 2E). These data indicate that SNHG16 may interact with miR-302a-3p and depress its expression in HCC.

SNHG16/miR-302a-3p axis regulates the expression of FGF19 in liver cancer cells. To further search the novel role of SNHG16/miR-302a-3p pathway in liver cancer cells, we identified potential targets of miR-302a-3p using TargetScan and MiRanda tools and found miR-302a-3p recognition site in the 3'-UTR of FGF19 (Figure 3A), and the interaction was validated via luciferase assay (Figure 3B). As FGF19 
A

\begin{tabular}{|c|c|c|}
\hline SNHG16 (Mut) & 5'- ${ }^{C A C}{ }_{G G U}{ }^{C} \cup U^{C} G U A^{U} C$ & ${ }_{\text {TCGTGAA }}^{\text {CUUU }}$ \\
\hline SNHG16 (WT) & 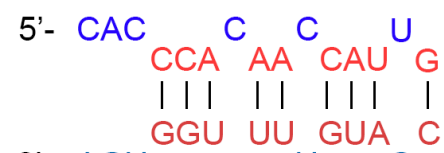 & $\begin{array}{l}\text { CUUU } \\
\text { AGCACUU }{ }^{-3} \\
|||||||| \mid \\
\text { UCGUGAA }\end{array}$ \\
\hline
\end{tabular}

B

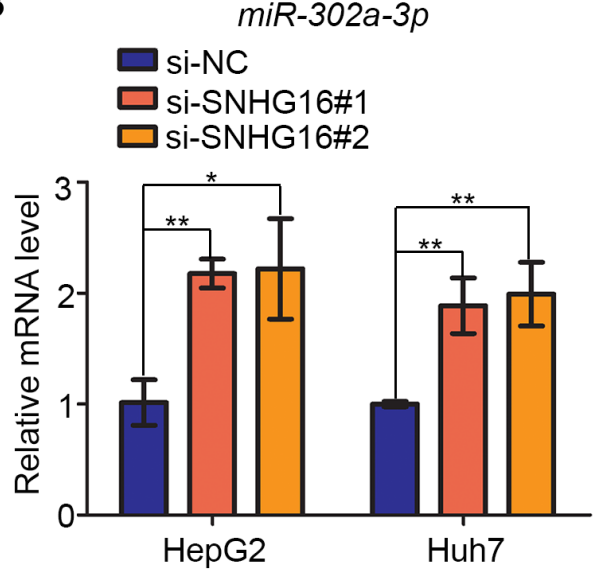

D
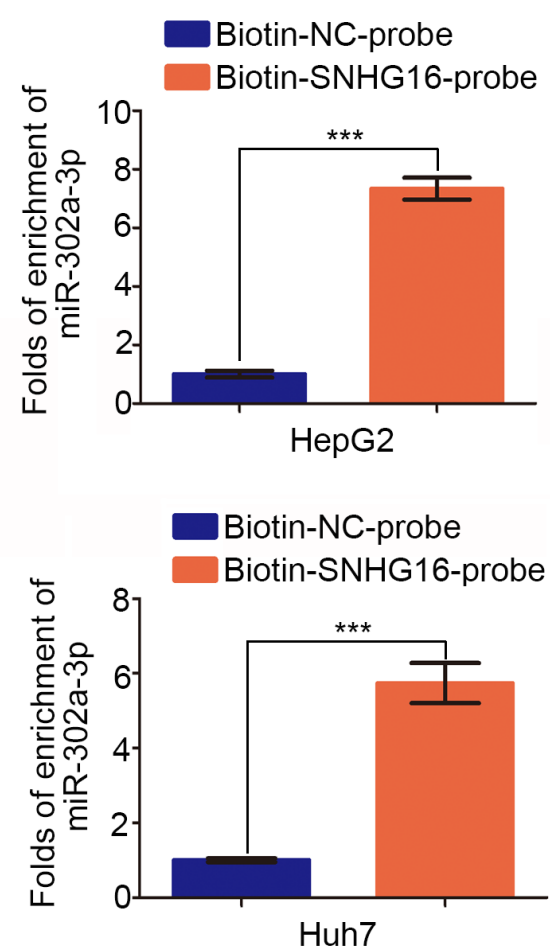

C

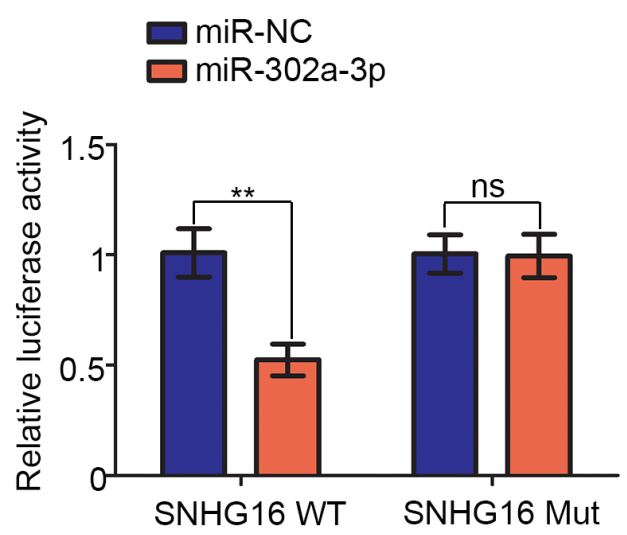

E

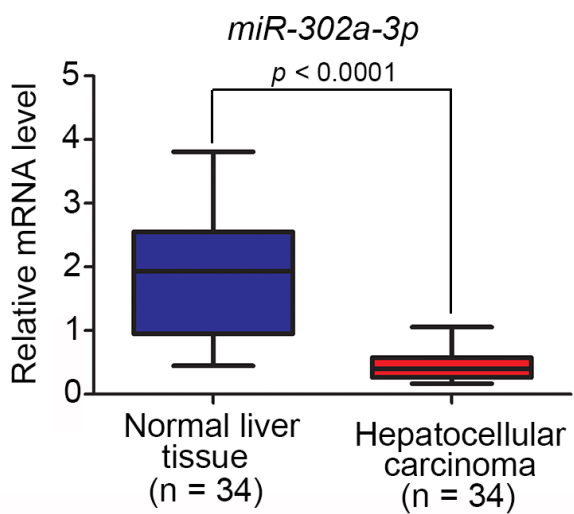

$\mathbf{F}$

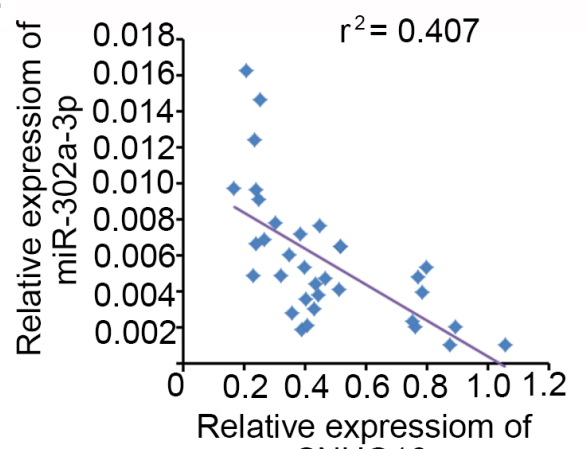

SNHG16

Figure 2. SNHG16 interacts with miR-302a-3p and depresses its expression in HCC. A) The predicted position of miR-302a-3p binding sites on the SNHG16 transcript. B) HepG2 and Huh7 cells were transfected with indicated constructs. 48h post transfection, cells were harvested for RT-qPCR analysis. Data shown are mean values \pm SD from three replicates. ${ }^{*} p<0.05 ;{ }^{* *} p<0.01$. C) HepG2 cells were transfected with SNHG16 (WT) or SNHG16 (Mut) reporter and miR-NC or miR-302a-3p mimic. After $48 \mathrm{~h}$, relative luciferase activity was detected using a dual-luciferase reporter assay. D) HepG2 and Huh7 cells were transfected with indicated constructs. $48 \mathrm{~h}$ post transfection, cells were harvested for RT-qPCR analysis. Data shown are mean values $\pm S D$ from three replicates. ${ }^{* *} p<0.001$. E) miR-302a-3p expression was downregulated in liver cancer tissues compared with normal liver tissue ( $(n=34)$. F) A negative correlation between SNHG16 expression and miR-302a-3p expression in liver cancer tissues. 
A

\section{Position 880-886 of FGF19 3'UTR}

FGF19 WT 5'...UAGAACCCUUUCCCCAGCACUUG...3' | | | | |

miR-302a-3p 3'AGUGGUUUUGUACCUUCGUGAAU5'

FGF19 Mut 5'...UAGCCAAAGGUCCCCAGCACUUG...3'

B

miR-NC $\square$ miR-302a-3p mimic

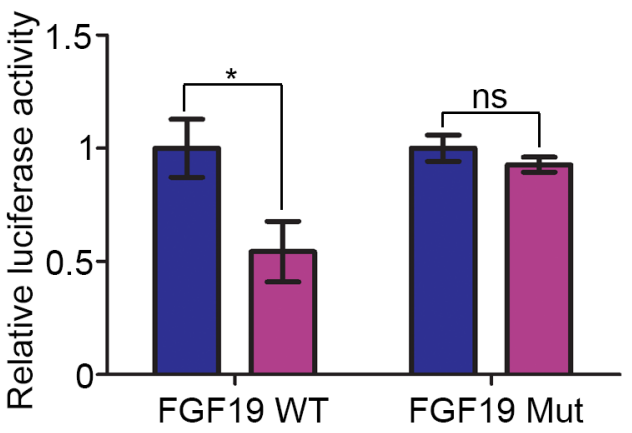

C

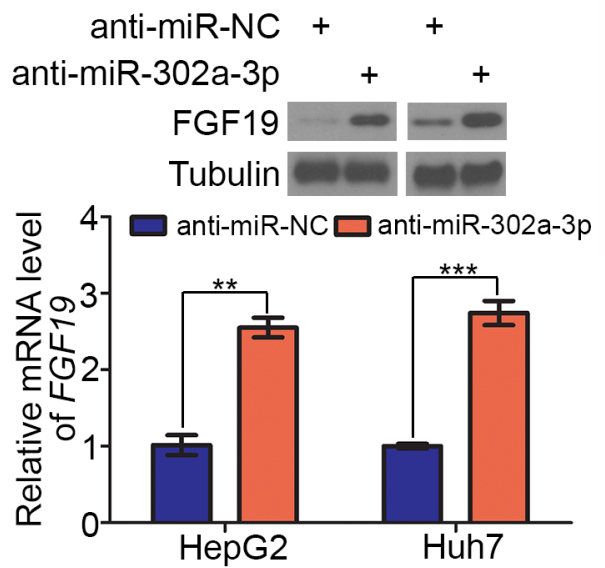

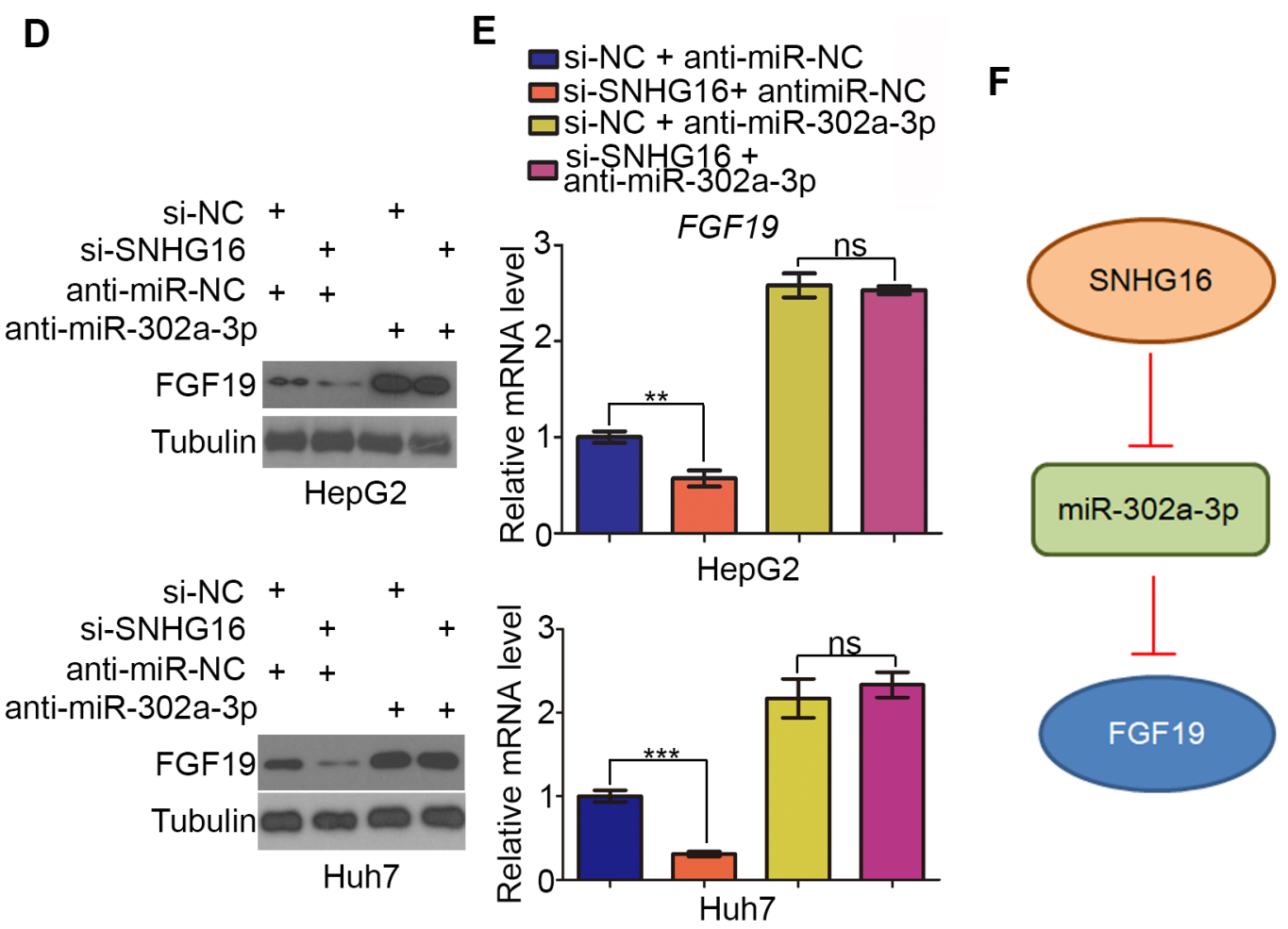

Figure 3. SNHG16/miR-302a-3p axis regulates the expression of FGF19 in liver cancer cells. A) The predicted position of miR-302a-3p binding sites on the FGF19 3'-UTR. B) HepG2 cells were transfected with FGF19 (WT) or FGF19 (Mut) reporter and miR-NC or miR-302a-3p mimic. After 48 h, relative luciferase activity was detected using a dual-luciferase reporter assay. C) HepG2 and Huh7 cells were transfected with indicated constructs. 48h post transfection, cells were harvested for western blotting and RT-qPCR analysis. Data shown are mean values \pm SD from three replicates. ${ }^{* *} \mathbf{p}<0.01$; ${ }_{* * *} \mathrm{p}<0.001$. HepG2 and Huh7 cells were transfected with indicated constructs. $48 \mathrm{~h}$ post transfection, cells were harvested for western blotting (D) and RT-qPCR analysis (E). Data shown are mean values \pm SD from three replicates. ns, not significant; ${ }^{* *} p<0.01$. F) A hypothetical model depicting that SNHG16 binds and represses the expression of miR-302a-3p, and FGF19 is the target of miR-302a-3p, thus SNHG16/miR-302a-3p axis regulates the expression of FGF19 in liver cancer cells. 
A

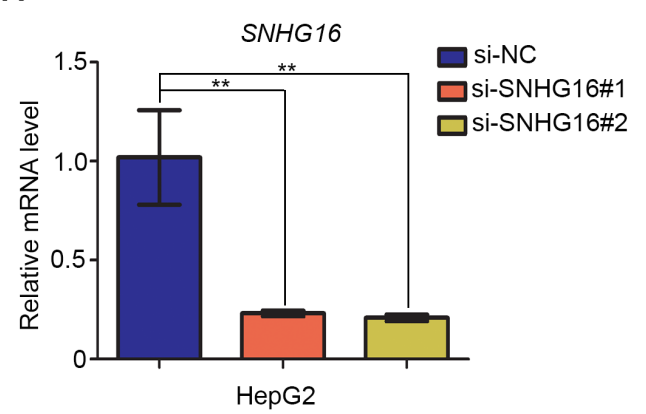

C

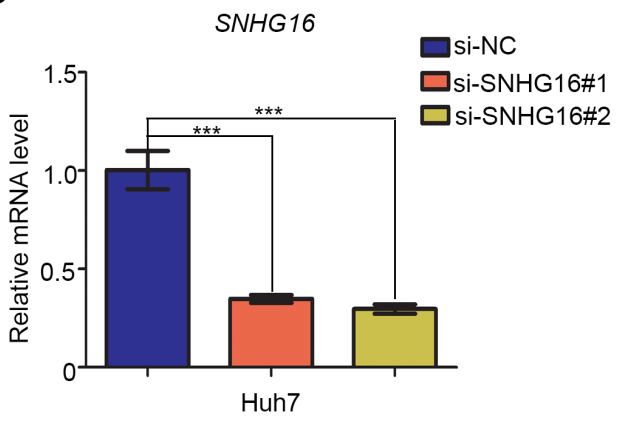

E

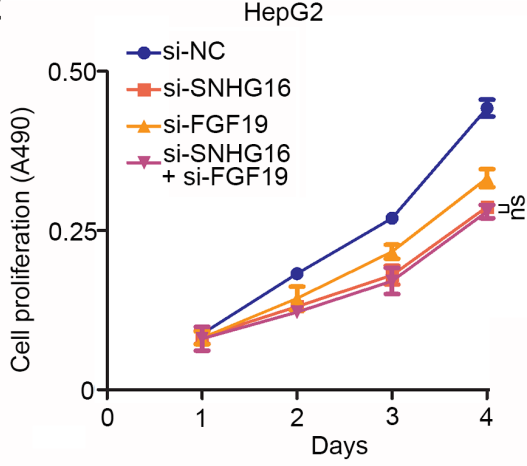

B

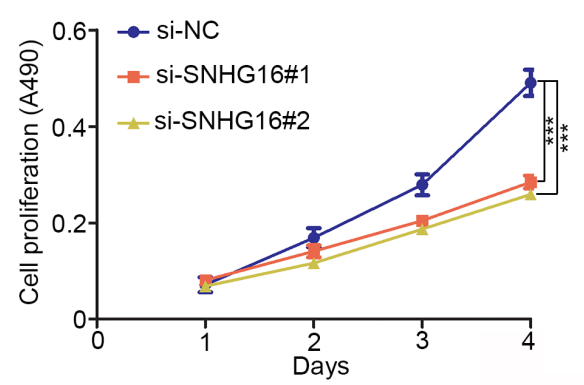

D

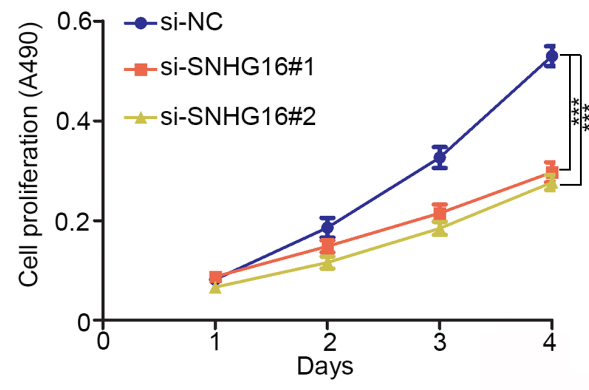

Huh7

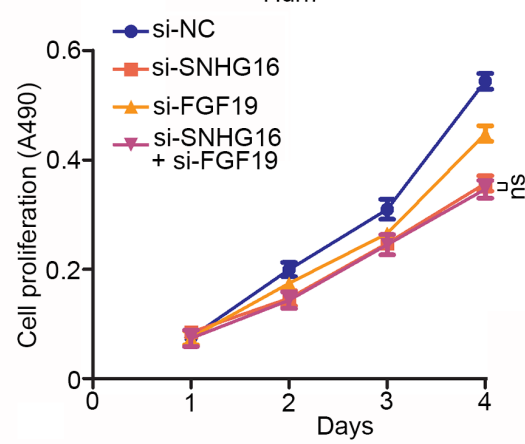

$\mathbf{F}$

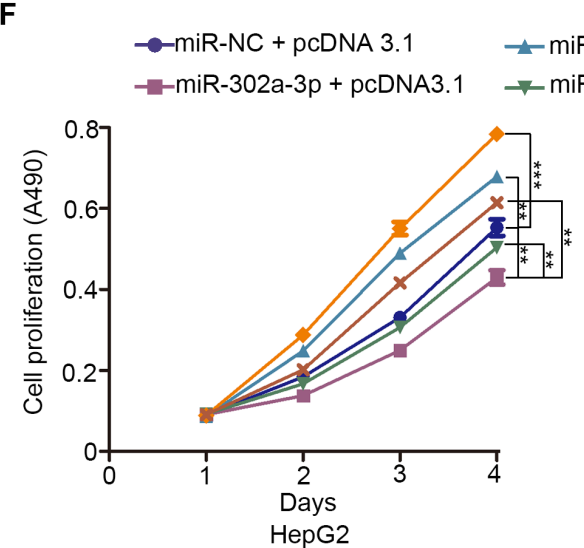

$$
\begin{aligned}
& \neq \text { miR-NC + FGF19 } \quad \leadsto \text {-miR-NC + SNHG16 } \\
& \text { *-miR-302a-3p + SNHG16 }
\end{aligned}
$$

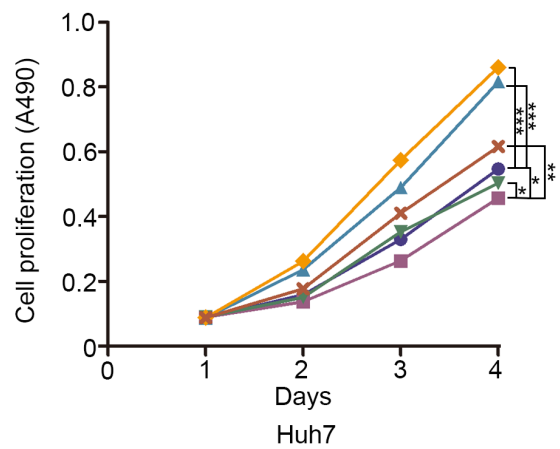

Figure 4. SNHG16 promotes cell proliferation via SNHG16/ miR-302a-3p/FGF19 axis. HepG2 (A) and Huh7 (C) cells were transfected with indicated constructs. $48 \mathrm{~h}$ post transfection, cells were harvested for RT-qPCR analysis (A and C) and MTS assay (B and D). For RT-qPCR analysis ,data shown are mean values \pm SD from three replicates. ${ }^{* *} \mathrm{p}<0.01 ;{ }^{* * *} \mathrm{p}<0.001$. For MTS assay, data shown are mean values \pm SD from five replicates. ${ }^{* *} p<0.01$; ${ }_{* * *} \mathbf{p}<0.001$. E) HepG2 and Huh7 cells were transfected with indicated constructs. $48 \mathrm{~h}$ post transfection, cells were harvested for MTS assay. Data shown are mean values \pm SD from five replicates. ns, not significant. F) HepG2 and Huh7 cells were transfected with indicated constructs. 48h post transfection, cells were harvested for MTS assay. Data shown are mean values \pm SD from five replicates. ns, not significant. ${ }^{*} \mathbf{p}<0.05 ;{ }^{* *} \mathbf{p}<0.01 ;{ }^{* * *} \mathbf{p}<0.001$. 
acting as an oncogenic driver in HCC [10], we want to verify whether SNHG16/miR-302a-3p axis regulated the expression of FGF19. As expected, the FGF19 expression was up-regulated after treated anti-miR-302a-3p in HepG2 and Huh7 cells (Figure 3C). Moreover, we showed that knockdown of SNHG16 decreased the expression of FGF19 and this effect was diminished after treated with anti-miR302a-3p in HepG2 and Huh7 cells (Figures 3D, 3E). These results suggest that FGF19 is a direct target of miR-302a-3p and SNHG16/miR-302a-3p axis regulates the expression of FGF19 in liver cancer cells (Figure 3F).

SNHG16 promotes cell proliferation via SNHG16/ miR-302a-3p/FGF19 axis. Our previous finding indicated that SNHG16 was overexpressed and associated with poor prognosis in hepatocellular carcinoma. To explore the function of SNHG16 in liver cancer progression, we knocked down SNHG16 in both HepG2 and Huh7 cells. MTS assay showed that knockdown of SNHG16 inhibits cell growth rate in liver cancer cells (Figures 4A-4D). Furthermore, we showed that knockdown of FGF19 also decrease the cell proliferation rate of liver cancer cells (Figure 4E). However, co-knockdown of FGF19 and SNHG16 could inhibit cell growth more than knockdown of SNHG16 alone (Figure 4E). Moreover, we also showed that miR-302a-3p inhibits the tumor growth and this effect could be rescued by overexpression of FGF19 or SNHG16 in both HepG2 and Huh7 cells (Figure 4F). These results indicate that SNHG16 inhibition significantly decreases liver cancer cell proliferation, and this process may mediate via SNHG16/ miR-302a-3p/FGF19 axis in HCC.

\section{Discussion}

It has been documented that aberrant expression of lncRNAs is common in human solid tumors and critical for tumor progression [11]. In our study, we demonstrated that lncRNA SNHG16 was not only significantly increased in HCC patient samples but also in liver cancer cell lines. Furthermore, up-regulated SNHG16 was linked to poor prognosis in HCC patients. Those findings indicate that SNHG16 is involved in liver cancer progression.

MicroRNAs (miRNAs) are type of non-coding RNAs that can regulate a set of target genes through translational repression or mRNA degradation $[12,13]$. The miR-302a-3p is one of the miR-302-367 cluster members [14]. It has been reported that miR-302s inhibits CDK4 and CCND1 expression and regulates cell cycle $[15,16]$. Moreover, the miR-302s decreased cancer cell proliferation by down-regulating the SDC1, SOX1 and SHH [17]. Thus, miR-302s showed tumor suppressive activity in a number of solid tumors. It has been reported that lncRNAs might compete with miRNAs as miRNA sponges to depress its expression [18]. In our present study, we show that SNHG16 targets miR-302a-3p and represses its expression in HCC.

FGF19 is a metabolic regulator gene belonging to the hormone-like FGF family $[19,20]$. Recent studies demon- strate that FGF19 acts as an oncogene by specifically binding to FGFR4 and activating various intracellular pathways, including GSK3 $\beta / \beta$-catenin/E-cadherin signaling [21]. FGF19 promotes tumor progression in liver cancer cells and positively correlates with poor prognosis in HCC patients. Moreover, FGF19/FGFR4 signaling contributes to the resistance of hepatocellular carcinoma to sorafenib [10]. Therefore, FGF19 is an ideal target for HCC therapy. In our study, we demonstrate that FGF19 is a bona fide target gene of miR-302a-3p in HCC. Furthermore, our findings indicate that SNHG16/miR-302a-3p-mediated expression of FGF19 regulates malignant behavior of liver cancer cells.

Taken together, our study demonstrates that lncRNA SNHG16 is overexpressed in HCC and associated with poor prognosis in HCC patients. SNHG16 repressed expression of miR-302a-3p in HCC. Moreover, miR-302a-3p decreased FGF19 expression in liver cancer cells. Therefore, SNHG16 regulated cancer cell progression through SNHG16/ miR-302a-3p axis in HCC, which suggested that SNHG16 might be a predictive biomarker and a potential therapeutic target of liver cancer.

\section{References}

[1] LIU N, LIU Q, YANG X, ZHANG F, LI X et al. Hepatitis B virus-upregulated lnc-HUR1 promotes cell proliferation and tumorigenesis by blocking p53 activity. Hepatology 2018; 68: 2130-2144. https://doi.org/10.1002/hep.30098

[2] DING B, LOU W, XU L, FAN W. Non-coding RNA in drug resistance of hepatocellular carcinoma. Biosci Rep 2018; 38. https://doi.org/10.1042/BSR20180915

[3] CORRA F, AGNOLETTO C, MINOTTI L, BALDASSARI F, VOLINIA S. The Network of Non-coding RNAs in Cancer Drug Resistance. Front Oncol 2018; 8: 327. https://doi. org/10.3389/fonc.2018.00327

[4] YU M, OHIRA M, LI Y, NIIZUMA H, OO ML et al. High expression of ncRAN, a novel non-coding RNA mapped to chromosome 17q25.1, is associated with poor prognosis in neuroblastoma. Int J Oncol 2009; 34: 931-938. https://doi. org/10.3892/ijo_00000219

[5] ZHU Y, YU M, LI Z, KONG C, BI J et al. ncRAN, a newly identified long noncoding RNA, enhances human bladder tumor growth, invasion, and survival. Urology 2011; 77: 510. e1-5. https://doi.org/10.1016/j.urology.2010.09.022

[6] CHRISTENSEN LL, TRUE K, HAMILTON MP, NIELSEN MM, DAMAS ND et al. SNHG16 is regulated by the Wnt pathway in colorectal cancer and affects genes involved in lipid metabolism. Mol Oncol 2016; 10: 1266-1282. https:// doi.org/10.1016/j.molonc.2016.06.003

[7] TANG Z, LI C, KANG B, GAO G, LI C et al. GEPIA: a web server for cancer and normal gene expression profiling and interactive analyses. Nucleic Acids Res 2017; 45: W98W102, 2017. https://doi.org/10.1093/nar/gkx247

[8] KARTHA RV, SUBRAMANIAN S. Competing endogenous RNAs (ceRNAs): new entrants to the intricacies of gene regulation. Front Genet 2014; 5: 8. https://doi.org/10.3389/ fgene. 2014.00008 
[9] LI JH, LIU S, ZHOU H, QU LH, YANG JH. starBase v2.0: decoding miRNA-ceRNA, miRNA-ncRNA and proteinRNA interaction networks from large-scale CLIP-Seq data. Nucleic Acids Res 2014; 42: D92-97. https://doi.org/10.1093/ nar/gkt1248

[10] GAO L, WANG X, TANG Y, HUANG S, HU CA et al. FGF19/FGFR4 signaling contributes to the resistance of hepatocellular carcinoma to sorafenib. J Exp Clin Cancer Res 2017; 36: 8. https://doi.org/10.1186/s13046-016-0478-9

[11] SLABY O, LAGA R, SEDLACEK O. Therapeutic targeting of non-coding RNAs in cancer. Biochem J 2017; 474: 42194251. https://doi.org/10.1042/BCJ20170079

[12] CHEN E, XU X, LIU R, LIU T. Small but Heavy Role: MicroRNAs in Hepatocellular Carcinoma Progression. Biomed Res Int 2018; 2018: 6784607. https://doi. org/10.1155/2018/6784607

[13] WONG CM, TSANG FH, NG IO. Non-coding RNAs in hepatocellular carcinoma: molecular functions and pathological implications. Nat Rev Gastroenterol Hepatol 2018; 15: 137-151. https://doi.org/10.1038/nrgastro.2017.169

[14] GAO Z, ZHU X, DOU Y. The miR-302/367 cluster: a comprehensive update on its evolution and functions. Open Biol 2015; 5: 150138. https://doi.org/10.1098/rsob.150138

[15] LIN SL, CHANG DC, YING SY, LEU D, WU DT. MicroRNA miR-302 inhibits the tumorigenecity of human pluripotent stem cells by coordinate suppression of the CDK2 and CDK4/6 cell cycle pathways. Cancer Res 2010; 70: 94739482. https://doi.org/10.1158/0008-5472.CAN-10-2746
[16] CARD DA, HEBBAR PB, LI L, TROTTER KW, KOMATSU $\mathrm{Y}$ et al. Oct4/Sox2-regulated miR-302 targets cyclin D1 in human embryonic stem cells. Mol Cell Biol 2008; 28: 64266438. https://doi.org/10.1128/MCB.00359-08

[17] LI Y, HUO J, PAN X, WANG C, MA X. MicroRNA 302b$3 p / 302 c-3 p / 302 d-3 p$ inhibits epithelial-mesenchymal transition and promotes apoptosis in human endometrial carcinoma cells. Onco Targets Ther 2018; 11: 1275-1284. https:// doi.org/10.2147/OTT.S154517

[18] THOMSON DW, DINGER ME. Endogenous microRNA sponges: evidence and controversy. Nat Rev Genet 2016; 17: 272-283. https://doi.org/10.1038/nrg.2016.20

[19] FENG S, DAKHOVA O, CREIGHTON CJ, ITTMANN M. Endocrine fibroblast growth factor FGF19 promotes prostate cancer progression. Cancer Res 2013; 73: 2551-2562. https:// doi.org/10.1158/0008-5472.CAN-12-4108

[20] SAWEY ET, CHANRION M, CAI C, WU G, ZHANG J et al. S. Identification of a therapeutic strategy targeting amplified FGF19 in liver cancer by Oncogenomic screening. Cancer Cell 2011; 19: 347-358. https://doi.org/10.1016/j. ccr.2011.01.040

[21] Repana D, Ross P. Targeting FGF19/FGFR4 Pathway: A Novel Therapeutic Strategy for Hepatocellular Carcinoma. Diseases 2015; 3: 294-305. https://doi.org/10.3390/diseases3040294 